\title{
The accretion disk paradigm for young stars
}

\section{Claude Bertout}

\author{
Institut d'Astrophysique, 98bis, Boulevard Arago, 75014 Paris, France
}

\begin{abstract}
Accretion and magnetic fields play major roles in several of the many models put forward to explain the properties of $\mathrm{T}$ Tauri stars since their discovery by Alfred Joy in the 1940s. Early investigators already recognized in the 1950s that a source of energy external to the star was needed to account for the emission properties of these stars in the optical range.

The opening of new spectral windows from the infrared to the ultraviolet in the 1970s and 1980s showed that the excess emission of $\mathrm{T}$ Tauri stars and related objects extends into all wavelength domains, while evidence of outflow and/or infall in their circumstellar medium was accumulating.

Although the disk hypothesis had been put forward by Merle Walker as early as 1972 to explain properties of YY Orionis stars and although Lynden-Bell and Pringle worked out the accretion disk model and applied it specifically to T Tauri stars in 1974, the prevailing model for young stellar objects until the mid-1980s assumed that they experienced extreme solar-type activity. It then took until the late 1980s before the indirect evidence of disks presented by several teams of researchers became so compelling that a paradigm shift occurred, leading to the current consensual picture.

I briefly review the various models proposed for explaining the properties of young stellar objects, from their discovery to the direct observations of circumstellar disks that have so elegantly confirmed the nature of young stars. I will go on to discuss more modern issues concerning their accretion disk properties and conclude with some results obtained in a recent attempt to better understand the evolution of Taurus-Auriga young stellar objects.
\end{abstract}

Keywords. Stars: formation, stars: pre-main sequence.

\section{Introduction}

Like many topics in astrophysical research, the idea that stars might be evolving bodies that form from diffuse gas was first dreamt of by great ancient minds. The 18th century scientist Pierre-Simon de Laplace already thought that the Sun and planets coalesced from a flattened and rotating gaseous nebula under the action of gravity (Marquis de Laplace 1798). The idea was reborn in the mid- $20^{\text {th }}$ century when the theory of stellar evolution emerged and in less than one generation, evolved into a full-fledged, highly successful research area that grew very quickly and branched into several more specialized subfields. This symposium, devoted to one of these subfields of active topical research, investigates the role of both a magnetic field and accretion in shaping the properties of young stellar objects.

It is amusing, but also revealing of the scientific approach, that these two physical processes were already invoked in the late 1940s for explaining some properties of young stars even before it became apparent that they were young. There is only a relatively small number of basic physical phenomena that can be called upon to explain the properties of celestial bodies. If one proceeds by elimination in a logical and careful way to restrict the range of possible mechanisms that could explain an observed situation, there is a good chance that one will correctly identify a plausible physical basis for any phenomenon. Laplace's conclusion that gravity was at work in shaping the primitive solar nebula proceeded from such a line of thought. 
The devil, however, is in the details. There is a long path and many dead ends from such an initial intuition to a consensual paradigm explaining a wide range of observational properties. The next section recalls some of the steps that researchers have gone through over the last 60 years to establish a working paradigm for young stars; because of space limitations, I will focus mainly on T Tauri stars (TTSs hereafter). I then briefly discuss some of the current open issues concerning disk properties.

\section{Historical notes}

Alfred H. Joy (1882-1973) is remembered for his observational contributions to the field of stellar spectroscopy, beginning with the Mount Wilson radial velocity and spectroscopic parallax program, which led in 1935 to the first comprehensive HertzsprungRussell diagram with 4179 stars (Adams et al. 1935), followed by his pioneering studies of long-period regular and semi-regular variables (e.g., Joy 1952, 1954) and his involvement with late-type erratic variables ( e.g., the first observation of a flare in UV Cet by Joy \& Humason 1949; Joy 1958). However, as George Herbig (1974) pointed out, "It is difficult to be certain which was Joy's most far-reaching contribution, but the T Tauri stars are his most famous monument. In the early 1950s it was becoming painfully clear that given the sources of stellar energy, star formation must be a continuing thing, presumably going on before our eyes [...] Several years passed before it came to be recognized generally that the link [between stars and the interstellar clouds] existed, described in that 1945 investigation by Joy. [...] Twenty years later, the dimensions of that discovery are just beginning to be appreciated".

The paper that George Herbig refers to is, of course, the now classical work that defined the class of "T Tauri Variable Stars" (Joy 1945). This work was soon followed by additional discoveries of TTSs in Taurus (Joy 1949) and Ophiuchus (Struve \& Rudkjøbing 1949). The criteria given by Joy to define the class of TTSs were

(a) "irregular light variations of about $3 \mathrm{mag}$,

(b) spectral type F5-G5 with emission lines resembling the solar chromosphere,

(c) low luminosity,

(d) association with dark or bright nebulosity".

Seventeen years later, Herbig (1962) gave a purely spectroscopic definition of the class, using the following criteria:

(a) "The hydrogen lines and the lines of CaII are in emission.

(b) The fluorescent FeI emission lines $\lambda \lambda$ 4063, 4132 are present (they have been found only in T Tauri stars).

(c) the [SiII] emission lines $\lambda \lambda$ 4068, 4076 are usually but not always present. Probably [SII] $\lambda \lambda 6717,6731$ and [OI] $\lambda \lambda 6300,6363$ are also characteristic.

(d) Recent results [...] suggest that the presence of strong LiI $\lambda 6707$ absorption, in those stars in which an absorption spectrum can be seen at all, may constitute another primary criterion".

Herbig also notes that the emission lines are usually superimposed on a continuous spectrum ranging from an approximately normal absorption spectrum in the range $\mathrm{F}$ to $\mathrm{M}$ to pure continuous emission, with all intermediary stages in between. Herbig's spectroscopic characteristics define what we now call the classical T Tauri stars (CTTSs hereafter). When all present in the same stars, these criteria signal one of the most active stars of the class. Less active CTTSs do not show FeI fluorescent emission or forbidden emission, but all have strong Balmer and CaII H and K line emission, as well as strong LiI absorption, a feature now known to be the main signature of their youth. 
The "veiling" continuous emission that obliterates the late-type absorption spectrum of CTTSs had already been noted by Joy and was considered as early as 1950 as a strong hint that a non-stellar emission component contributes to the blue part of the CTTS spectra (Greenstein 1950). In fact, Greenstein anticipated much of the controversy that would develop around CTTSs in the following decades when he wrote that "The veiling continuum and the emission lines must be produced in a circumstellar fringe or extended chromosphere".

\subsection{0 s and 1950s: first TTS models}

The first model for TTSs that emerged shortly after their discovery was motivated by their association with dark nebulae. These stars, postulated e.g. Struve \& Rudkjøbing (1949), might be normal field stars that were crossing and interacting with interstellar clouds. However, it quickly became clear that the kinetic energy of the gas falling onto the star was not high enough to account for the observed line and excess continuum luminosity; assuming a differential velocity of $10 \mathrm{~km} / \mathrm{s}$ between gas cloud and star and a cloud density of $10^{-18} \mathrm{~g} / \mathrm{cm}^{3}$, Greenstein (1950) derived a kinetic luminosity of a few $10^{-5} L_{\odot}$, whereas the observed excess luminosity can be greater than $L_{\odot}$ in extreme CTTSs. Even though the value of a typical CTTS luminosity excess was unknown in the early 1950s, it was already clear that it was orders of magnitude larger than what the accretion of interstellar cloud gas could provide. Faced with this problem, Greenstein (1950) thus proposed a variant of this model in which the (then newly discovered) magnetic field of the interstellar matter falling onto the star acted to compress and heat the infalling matter and led to the propagation of Alfvén waves in the stellar surroundings that could be responsible for the widths of several hundreds of $\mathrm{km} / \mathrm{s}$ observed in CTTS Balmer lines. Greenstein (1950) went on to speculate that "the incoming material apparently does not itself carry sufficient energy to the star, but it seems well suited as a source of the high kinetic temperature and as a trigger mechanism for the release of excess energy from star spots, in the form of flares bright enough to affect the total spectrum of the object".

It is therefore clear that the two physical processes, accretion and magnetism - the topic of today's symposium because we have strong evidence that they are responsible for young star activity - had already been identified as such as early as in 1950. Unfortunately, as data started to accumulate, these promising physical findings were clouded by what appeared to be contradictory observations, and it took 40 years to disentangle the various issues and understand "the nature of the objects of Joy" $\dagger$.

What was less evident in the late 1940s and early 1950s was the evolutionary status of TTSs. Although Ambartsumian (1947, 1954) introduced the concept of stellar OB and $\mathrm{T}$ associations and proposed that they were the sites of ongoing star formation shortly after TTSs were discovered, these ideas were long in gaining mainstream acceptance in the community, presumably due to the difficulties of communication between the Eastern the Western worlds after World War II. The first paradigm shift, from stars passing by chance through interstellar clouds to stars being born in interstellar clouds, took place around 1955 and was strongly influenced by Ambartsumian's ideas.

The state of research on TTSs in the mid-50s is summarized in the proceedings of the IAU Symposium No.3 "Non-stable stars" that took place in Dublin in September 1955. There, Herbig (1957) discussed the nature of TTSs, whose pre-main sequence status appears to be by then an accepted fact, but it is their relationship to the newly discovered Herbig-Haro objects (Herbig 1951; Haro 1952) that takes the center stage in Herbig's

$\dagger$ This cute expression is the title of a paper by Rydgren et al. (1976) that fails to disclose the true nature of these objects but remains a strong contribution to our knowledge of their infrared properties. 
talk, which gives arguments based in part on the association of Burnham's nebula with $\mathrm{T}$ Tau itself, supporting the view that the nebulous Herbig-Haro objects might be the precursors of TTSs. Another subject of discussion during the symposium was the relationship between flare stars and TTSs. This interest was motivated by Guillermo Haro having discovered a number of flare stars (which he called flash stars to distinguish them from UV Cet stars) in Taurus and Orion. In his talk, Haro (1957) concluded that TTSs and UV Cet stars must have a similar nature. Alfred Joy, on the other hand, reviewed the properties of the three classes of late-type variables, the TTSs, the UV Cet stars, and the first examples of cataclysmic variables and wisely concluded: "Somewhat similar effects are noted in the spectral changes of the three groups of stars, but the underlying cause of the strange and unexpected behaviour, which may be quite different in the three types, invites both observations and analysis" (Joy 1957). In contrast, the somewhat similar spectral properties of TTSs, flare stars, Herbig-Haro objects and cataclysmic variables appeared so unusual to Ambartsumian that he felt compelled to propose that they were due to the liberation of large amounts of energy connected with nuclear processes but different from the already known thermo-nuclear reactions. He went on to add: "It may be that the material being brought up from the interior contains pre-stellar matter of high density. It may represent matter that is in an altogether peculiar state, thus far unknown to us. [...] the rapidly growing knowledge of T Tauri stars permits us to pass on to the study of the laws of physical processes of a new type, which govern a number of phenomena taking place in these stars" (Ambartsumian 1957). Clearly, understanding the origin of the energy input needed to explain the exotic properties of TTSs had already become a true challenge for astrophysicists, and some bold ideas were emerging. Reacting to this suggestion, Burbidge \& Burbidge (1955) emphasized that the energy sources responsible for flare activity must have different natures in different classes of stars and that no new physical mechanism was needed to understand them. They wrote "To sum up, we wish to divide the stars showing flaring into two main categories: (a) The $\mathrm{T}$ Tauri and related objects in which MHD processes outside the star are responsible. (b) The dMe flare stars in which internal MHD processes are responsible". By the end of the 1950s, then, the main physical question concerning these stars was on the table: were the radiative energy losses observed in TTSs covered by the star itself or by some external energy source?

\subsection{0s: evidence for T Tauri winds}

The first major review of the T Tauri phenomenon by Herbig (1962) and Leonard Kuhi's thesis work (Kuhi 1964) were the milestones of the first half of the 1960s and a major evolution in ideas on the envelopes of T Tauri stars. Herbig reviewed the scarce highresolution spectroscopic data and concluded that "mass ejection by T Tauri stars [...] seems to be such a common trait of the family that it must find some natural explanation in the contractional process", while Kuhi convincingly demonstrated that the $\mathrm{H}_{\alpha}$ line profiles of several bright stars of the class could be modeled by a spherical wind. Derived mass-loss rates were in the $10^{-8}-10^{-7} M_{\odot} / \mathrm{yr}$, and this work became the first of numerous attempts to model the line profiles of young stars. Another result obtained by Kuhi attracted much less attention, although it was crucial. Namely, Kuhi (1966) found that the UV excess of TTSs is correlated with $\mathrm{H}_{\alpha}$ intensity, making it apparent that the UV excess was due to Balmer continuum emission. This finding opened the door to a model where, by analogy with the Sun, one postulates that the T Tauri chromospheres could possibly be denser than in the Sun and could thus give rise to the UV excess.

Meanwhile, a few doors away from Herbig's office at Lick Observatory, Merle F. Walker was studying faint peculiar stars in Orion that showed particularly strong UV excesses. 
He had been using a Lallemand electronic camera to obtain flux-calibrated spectrograms of a number of these objects, now known as YY Ori stars after their prototype, and had noticed the presence of red-displaced, highly variable absorption components in the Balmer lines of these objects, indicating infall velocity of up to $400 \mathrm{~km} / \mathrm{s}$. In these spectra the Balmer jump is clearly in emission, and the overall line spectra look very much like those of usual TTSs. Walker (1963) suggested that "the presence of the excess continuous emission may have some connection with this possible infall of material", a prophetic statement that, unfortunately, no one paid attention to.

In the late 1960s, another major discovery was made by Eugenio E. Mendoza V. (1966, 1968) when he found that several TTSs and related objects had strong infrared excesses up to $5 \mu \mathrm{m}$. Mendoza, who was using Johnson's photometric equipment at the Lunar and Planetary Laboratory, also confirmed the UV excess and was the first to present quasi-simultaneous UBVRIJHKLM spectral energy distributions for a few bright young stars (T Tau, SU Aur, RW Aur, V380 Ori, and R Mon). By the end of the 1960s, major pieces of the T Tauri puzzle were known: their optical line spectra, their UV and IR excesses, their "veiling", their outflows and sometimes inflows. The energetics problem had become much more acute when the IR properties of young stars were discovered, but by then this question was set aside as specialists were focusing on trying to understand the mass motions in the surroundings of young stars.

\subsection{0s: pioneers and the great confusion}

The 1970s were a very contrasted decade as far as T Tauri research was concerned. On one hand, there were a few pioneers who anticipated the nature of TTSs, but their work remained largely unnoticed. On the other hand, there was the rest of us, who were more and more puzzled and confused by the data that we kept accumulating.

Meet the pioneers: observer Merle F. Walker and theoreticians Donald Lynden-Bell and Jim Pringle. Walker (1972) postulated a circumstellar disk for explaining the spectroscopic properties of YY Ori stars. He noted "That we might expect the infall to occur in a preferential plane during the last stages of the collapse of the prestellar cloud is suggested by analogy with the solar system, where the coplanar orbits of the planets indicate that the presolar nebula must have collapsed to a plane during the formation of the system". It was, to the best of my knowledge, the first time that a circumstellar disk was proposed as an explanation for specific observed properties of young stars. In Walker's model, the UV and blue continua are formed in a shock front where the infalling matter reaches the stellar surface, and the Balmer line emission originates in the disk with the red-displaced absorption components being formed in that part of the disk seen in projection onto the star by the observer. While Walker also discussed the possibility of a spherically symmetric envelope around these stars, he clearly favors the disk model in particular because "the shock front does not cover the entire surface of the star, so that the stellar absorption spectrum will be visible, if it is sufficiently luminous compared to the blue continuum, regardless of the optical thickness of the infalling matter or the material at the shock front". Because envelope modeling efforts started in the late 70s, it was not yet apparent to most researchers that the low visual extinction of most TTSs poses major difficulties for any model involving a spherically symmetric, dusty envelope. Here, Walker was providing an elegant solution to a problem that he was first to foresee, but unfortunately that solution had been forgotten a few years later when the problem actually did appear.

Lynden-Bell \& Pringle (1974) built a formidable theoretical foundation to the empirical disk picture envisioned by Walker. Their accretion disk model is such a powerful physical and mathematical construct that it underlies most of the work on circumstellar disks 
done ever since. While they applied their work specifically to TTSs, the community of young star specialists hardly noticed that Lynden-Bell and Pringle were offering an answer to many of their questions. Instead, it is in its application to interacting binaries that the accretion disk model, which had been independently worked out in that context by Shakura \& Sunyaev (1973), met with immediate success.

In 1976, researchers met for the IAU Symposium No. 75 entitled "Star formation", which was held in Geneva. Some emphasis was given during that conference to results of computations of protostellar collapse in spherical symmetry, since several groups had attempted to reproduce the pioneering numerical computations by Larson (1969). Relating these computations to observations of YY Ori and TTSs appeared difficult, although there were some hints that the IR spectral energy distribution of some bright TTSs bore some resemblance to theoretical spectra of the collapsing protostar models computed at the end of the accretion phase (cf. Larson 1977, and references therein). An amazingly upbeat review of young stellar object (YSO hereafter) properties was given on that occasion by Steve Strom (1977), who proposed in particular that most properties of TTSs could be explained by a dense gaseous spherical envelope at temperature $\approx 2 \cdot 10^{4} \mathrm{~K}$ and that Herbig-Haro objects were reflection nebulae illuminated by embedded stars. Strom mentioned briefly a new model for TTSs that was being worked out by Roger Ulrich (1976), who was able to reproduce the Type III P Cygni line profiles often observed in the $\mathrm{H}_{\alpha}$ line of TTSs in a model involving line emission from a shock front at the stellar surface. The shock wave is formed by the matter from an infalling and rotating protostellar envelope when it impacts the star.

The discussion following Strom's talk demonstrates the state of confusion that was prevalent among other observers of TTSs, with the contradictory evidence of infall and outflow obscuring most other issues. In his summary talk, Herbig (1977b) called for decisive observations and concluded:"After all, when the historians of science look back on our times with the perspective of the years, all that we do today will certainly be seen to have been either wrong, or irrelevant, or obvious". Thirty years later, this opinion appears to have been overly pessimistic; while some of the ideas discussed during that symposium were clearly wrong, none was irrelevant or particularly obvious. What remains stunning even today, however, is that during the Geneva symposium only two invited speakers mentioned, and only in passing, the Lynden-Bell \& Pringle (1974) accretion disk model; these reviewers were the two theoreticians Leon Mestel and Richard Larson.

We should not close this brief review of the 1970s without mentioning two extremely important pieces of work; Herbig (1977a) discussed observational evidence for FU Ori outbursts and their relationship to TTSs, while Cohen \& Kuhi (1979) presented their "magnum opus", an exhaustive discussion of TTS spectroscopic properties.

\subsection{0s: extension of the knowledge base and paradigm shift}

The first half of the 1980s saw an unprecedented increase in the available data on YSOs, owing to the launch of several orbiting observatories (IUE, IRAS, and the Einstein X-ray Observatory), as well as a strong increase in the efficiency of detectors used for groundbased observations in various wavelength domains. These technological advances led to a succession of discoveries, which are cited below in approximate chronological order.

- Snell et al. (1980) discovered the first instance of bipolar CO outflows, which turned out to be a ubiquitous phenomenon in starforming regions.

- Observations with the IUE satellite showed that the UV excess of TTSs extends to the far-UV and that the UV line spectrum is dominated by $\mathrm{Mg}$ II and includes transition region lines from ions such as Si II and C IV (e.g, Giampapa et al. 1981). 
- The Einstein X-ray Observatory revealed that typical TTS X-ray luminosities were within the $10^{30-31} \mathrm{erg} / \mathrm{s}$ range (e.g., Feigelson \& Decampli 1981), which ruled out spherical collapse as a possibility for explaining CTTS properties. Repeated observations also demonstrated the strong variability of TTSs in X-rays (Montmerle et al. 1983).

- The discovery of highly collimated jets shed new light on the nature of Herbig-Haro objects (Mundt \& Fried 1983).

- The association of Herbig-Haro objects with bipolar molecular outflows, discovered by Edwards \& Snell (1984), was crucial for understanding the nature of outflows emanating from YSOs.

- Mid-IR ground-based observations led to a new characterization of YSOs based on their IR properties (Lada \& Wilking 1984), thus paving the way for the work by Frank Shu and collaborators that led them to propose an evolutionary sequence from embedded protostellar sources to main sequence stars.

- IRAS observations showed that the IR excess of TTSs extends to the far-IR and that their spectral energy distribution (SED hereafter) is typically given by $\lambda F_{\lambda} \propto \lambda^{-1}$ (Rucinski 1985).

Meanwhile, theory was also moving forwards on several fronts. A first line of investigation was the deep chromosphere model advocated by Herbig and Kuhi. Detailed atmosphere models that included deep chromospheres were investigated, e.g., by Cram (1979) and Calvet et al. (1984). These models assumed that the chromospheric temperature minimum is located at a continuum optical depth $\tau_{c} \approx 0.1-0.3$ instead of $\tau_{c} \approx 10^{-3}$ $10^{-4}$ as in the Sun. Such a model accounts for the bulk properties of TTS emission line spectra and Balmer emission, but is unable to reproduce (a) the observed Balmer decrement, which indicates that the $\mathrm{H}_{\alpha}$ line originates from a more extended region, (b) the Balmer line shapes, which require high velocities of several hundred $\mathrm{km} / \mathrm{s}$ that are not present in the chromospheric region, and (c) the observed IR excess in the $1 \mu \mathrm{m}$ region, which remains optically thin in this atmospheric model where the main opacity source is the $\mathrm{H}^{-}$ion. Furthermore, this model ignores the energetics issue, which was emerging again after William De Campli (1981) discussed the energetic constraints involved in generating a strong $\mathrm{T}$ Tauri wind. He showed that the wind luminosity of a thermally driven wind with mass-loss rate $\approx 10^{-8} M_{\odot} /$ yr exceeds the stellar luminosity. De Campli went on to show that Alfvén-wave driven winds are more efficient and may account for moderately strong T Tauri winds. Detailed Alfvén-wave driven wind models were computed by De Campli (1981), Hartmann et al. (1982), and Lago (1984). Magnetic fields were also invoked to explain the photometric variability of TTSs, as Appenzeller \& Dearborn (1984) found from computations of pre-main sequence stellar structure models, that variable magnetic fields can cause visual brightness variations that are comparable in range (up to $3.5 \mathrm{mag}$ ) to what is observed in TTSs. It had therefore become evident in 1985 that a promising TTS model would combine a magnetically active star with a deep chromosphere and an Alfvén-wave driven wind. However, a model involving only the magnetic field had serious shortcomings in that it involved strong constraints on the stellar magnetic field strength and was unable to reproduce either the observed IR excess or the spectroscopic evidence for infall in some stars. Indeed, such a model was never developed because TTS research instead experienced a sudden paradigm shift with the comeback of the accretion disk model.

The return of the disk model for TTSs was announced by several precursor works. First, Martin Cohen (1983) suggested that the CTTS HL Tau was surrounded by a dusty disk. HL Tau has a "flat" SED in the IR (when plotted as $\log \lambda F_{\lambda}$ vs. $\log \lambda$ ), it is a TTS with both a $3.1 \mu \mathrm{m}$ ice feature and $10 \mu \mathrm{m}$ silicate absorption, and it displays a record linear polarization of $13 \%$. Cohen suggested that all these properties represent strong evidence 
of a circumstellar disk. Then, Appenzeller et al. (1984) showed that the forbidden lines of TTSs are often blue-displaced and can be interpreted as being formed in collimated outflows partially occulted by optically thick, dusty "screens", i.e., circumstellar disks. This first spectroscopic evidence of optically thick disks around TTSs was particularly convincing because the interpretation of optically thin forbidden line emission is much easier than that of permitted lines such as $\mathrm{H}_{\alpha}$. Finally, Hartmann \& Kenyon (1985) showed that FU Orionis outbursts can be explained as episodes of strong mass accretion from a disk. Emission from the hot, optically thick accretion disk dominates the system light at its maximum.

The IAU Symposium No. 122 "Circumstellar matter" held in Heidelberg in June 1986 can be seen as the moment when the disk hypothesis for TTSs re-emerged successfully on the public scene. Frank Shu presented there his ongoing work on the 4 stages of pre-main sequence evolution (Shu \& Adams 1987). Mundt (1987) discussed the large body of data concerning molecular outflows and optical jets, while Cohen (1987) discussed the possible role of disks in bipolar outflows observed in many different types of stars. Walter (1987) presented the properties of X-ray selected, "naked" TTSs that were to become known as weak-emission lines TTSs (WTTSs), i.e., TTSs that lack circumstellar disks. Meanwhile Finkenzeller \& Basri (1987) reported evidence of inflowing material in moderately active CTTSs. Finally, Bertout (1987) compared observed SEDs of TTSs with models of stars interacting with accretion disks.

The second half of the 1980s was marked by a wide exploration by several teams of the various consequences of the new paradigm, until accumulated evidence in favor of the accretion disk picture became so convincing that a consensus emerged concerning the presence of accretion disks around TTSs. The success of the model can be attributed not only to the fact that it solves many problems but also that it obeys lex parsimonice, also called Occam's razor rule. In other words, one hypothesis, the presence of a disk and its interaction with the star, accounts for most exotic properties of young stars. We briefly list in the following some influential works that led to the current consensus on disks and magnetospheric accretion. First, Frank Shu, Charlie Lada, and their collaborators developed an empirical/theoretical classification of YSOs based on the evolution of their SEDs and provided a global, physically consistent scheme for star formation (Adams et al. 1987; Shu et al. 1987). Rydgren \& Zak (1987) showed that the fraction of TTS system luminosity due to the IR-excess component is typically in the $0.4-0.8$ range and that the average mid-infrared spectral slope in their sample is $\lambda F_{\lambda} \propto \lambda^{-3 / 4}$. Kenyon \& Hartmann (1987), in an effort to explain this mid-IR slope, which is shallower than the law $\lambda F_{\lambda} \propto \lambda^{-4 / 3}$ expected for classical accretion disks, considered flaring, passive disks as an alternative to accretion disks. They also worked out detailed models of FU Ori disks (Hartmann \& Kenyon 1987). Edwards et al. (1987) extended the Appenzeller et al. (1984) study of forbidden line emission in TTSs and refined the forbidden line profile computation, confirming the need for an optically thick disk to explain these observations. Bertout et al. (1988) and Basri \& Bertout (1989) used quasi-simultaneous observations of TTSs in the UV, optical, and near-IR spectral ranges to compute detailed models of SEDs and derived mass accretion rates in the range $10^{-9}-10^{-7} M_{\odot} / \mathrm{yr}$ in the framework of the boundary layer model for the star-disk interaction. They also suggested, based on the observed rotational modulation of its UV and optical flux, that DF Tau was experiencing magnetospheric accretion. Adams et al. (1988) constructed models for TTSs with flat IR-spectra and were the first to suggest that gravitational instabilities might play a role in the accretion process. The first estimates of disk lifetimes were provided by Strom et al. (1989), while Cabrit et al. (1990) provided the first evidence for a connection between disk accretion and ejection of matter in a wind. Finally, 
Koenigl (1991) adapted the Ghosh \& Lamb (1979) magnetospheric accretion model to TTSs.

From 1990 on, the accretion disk paradigm was widely accepted and became the framework of "normal" T Tauri research. The body of theoretical results that has accumulated since then is very impressive and has led in particular to sophisticated $2 \mathrm{D}$ models of accretion disks (e.g., D'Alessio et al. 1998; Malbet et al. 2001; Dullemond et al. 2002; Pinte et al. 2006) that take into account in a self-consistent way such important processes as the vertical stratification of gas and dust in the disk, as well as some of the details of the magnetospheric accretion process (see the invited papers by S. Alencar, T. Harries, D. Ardila, and M. Romanova in this volume). Meanwhile, models of magnetized accretionejection structures, which were long limited to self-similar solutions due to the complexity of the MHD equations to be solved, are now becoming more realistic so that comparison with observations appears possible (see the reviews by S. Edwards, S. Cabrit, and F. $\mathrm{Shu}$ in this volume). On the observational side, one must of course mention that the presence of circumstellar disks in CTTSs has been confirmed by images from the Hubble Space Telescope and ground-based adaptive optics observations (e.g., Burrows et al. 1996; Close et al. 1997; Stapelfeldt et al. 1998, 2003). Today, near-IR interferometry allows us to probe the inner disk structure (see the review of F. Malbet in this volume). The next section provides a brief overview of some issues concerning basic disk properties that, in the opinion of this writer, remain open.

\section{Remarks on some open issues and a conclusion}

Basic properties of $\mathrm{T}$ Tauri disks that are still currently a matter of debate are the disk masses and mass-accretion rates. A number of methods have been used over the years to derive the mass-accretion rates from the optical spectra of large samples of CTTSs (e.g., Valenti et al. 1993; Hartigan et al. 1995; Gullbring et al. 1998). These estimates show that the stellar accretion rates of CTTSs are relatively well-known relative to each other, provided that they are determined using the same assumptions and methods; but rates determined with different methods can differ by a factor of 10 . Furthermore there is an uncertainty of typically a factor 3 on individual mass-accretion rates. The origin of these differences is understood fairly well (see Gullbring et al. 1998). The assumptions leading to these different sets of accretion rates differ mainly in the visual extinction values. While their relative validity can be discussed, none of the assumptions made by the various groups appears unreasonable. It should be emphasized, however, that the low mass-accretion rates derived by Gullbring et al. (1998) are lower limits to the true rates. These authors use the $J$ flux to estimate the photospheric luminosity and the visual extinction values that they adopt are particularly low; both assumption result in a low estimate for the accretion luminosity. A main reason for adopting these low massaccretion rates is because they provide for a consistent picture of disk evolution with the relatively low disk masses that were determined, e.g., by Beckwith \& Sargent (1991); a disk of mass $10^{-2} M_{\odot}$ provides an adequate reservoir for feeding a star during $10^{6}$ years at a rate of $10^{-8} M_{\odot} / \mathrm{yr}$.

Disk masses, however, are far from being well known and could be vastly underestimated. If there is appreciable grain growth and settling in the disk during the CTTS phase, resulting in the formation of a population of "sand and pebbles", as observations seem to indicate (cf. Natta et al. 2007), more mass than previously envisioned might be needed to reach the observed millimeter flux (Hartmann et al. 2006). There are other, more indirect lines of evidence that point in the direction of underestimated disk masses. It has been shown that the mass accretion rate depends on the stellar mass 
as $\dot{M} \propto M_{*}^{2.1}$, albeit with a large scatter (Muzerolle et al. 2003; Natta et al. 2005). As discussed by Alexander \& Armitage (2006) and Hartmann et al. (2006), a similar relationship between disk and stellar masses would be helpful for understanding the dependency of the mass accretion rate on the stellar mass. Another hint of higher disk masses comes from evolutionary aspects. In the current scheme of star formation, it is usually taken for granted that CTTSs evolve into WTTSs when their disk are fully accreted or dissipate for any other reason. The fact that CTTSs and WTTSs share the same region of the Hertzsprung-Russell diagram is usually interpreted as evidence of a large scatter in the properties of circumstellar accretion disks. While hard evidence for such an evolution was lacking until now, recent results provide support for this evolutionary scheme. Bertout et al. (2007) used the individual parallaxes computed for members of the Taurus-Auriga moving group by Bertout \& Genova (2006) to reassess the ages and masses of moving group members. They find that the age distributions of CTTSs and WTTSs are significantly different. Assuming that accretion of disk matter by the star proceeds at a constant rate and terminates when the disk is fully accreted, they find empirical evidence for evolution from CTTS to WTTS, provided that the disk mass is $\propto M_{*}^{2.85}$, leading to a disk lifetime of $\tau_{d}=4 \cdot 10^{6}\left(M_{*} / M_{\odot}\right)^{0.75}$. This surprising result implies very low masses for disks surrounding brown dwarfs unless the masses of CTTS disks have been underestimated. If confirmed, this result raises the question of the possible role of gravitational instabilities in driving the accretion process in these stars.

During this brief review of 60 years of T Tauri research, I have noted several instances of unfortunate "memory loss" when results obtained by one generation of scientists were forgotten, or ignored, by the next generation. Another example, observed in several recent papers, concerns the physical reason that led to the accretion disk hypothesis in the first place. The main physical problem that the disk hypothesis solves concerns the energetics of TTSs; the disk offers a reservoir of potential energy external to the star that can be tapped to power the phenomena associated with these stars (emission excesses at all wavelengths, jet and wind). A passive (non-accreting) disk only reprocesses the luminosity of the star and does not bring any gain as far as energetics of the system are concerned. While a fraction of the stellar luminosity is absorbed by the disk and re-radiated in the direction of the observer because of the system's anisotropy, this additional IR flux (compared to the same star but without a circumstellar disk) corresponds to at most $25 \%$ of the stellar luminosity in a flat disk (Adams \& Shu 1986) and up to $45 \%$ in a flaring disk (Kenyon \& Hartmann 1987), but there is no external energy source supplementing the star's in this model. TTSs with "classical" characteristics in the optical and UV spectral ranges (emission lines, veiling, UV excess, etc.) must therefore be surrounded by actively accreting disks rather than by passive disks.

The move from one generation of researchers to the next occurs on a timescale of one decade in modern research. Given the current pressure on young researchers to publish quickly and profusely, it is understandable that they have little time to find their own way in the maze of literature pertaining to previous research in their field, so as to gain a broad and unbiased view of their entire field of research before adding their own findings toward an even fuller picture. Above all other goals, I hope that this short review will offer some help in this formidable task.

\section{Acknowledgements}

I am deeply indebted to the scientific organizing committee for the dedication of this conference and for their kind and careful attention to avoiding the embarrassing moments that are often associated with such an honor. I commend the committee for organizing a clearly focused and very timely conference and gratefully acknowledge IAU support. 


\section{References}

Adams, F. C., Lada, C. J., \& Shu, F. H. 1987, ApJ, 312, 788

Adams, F. C. \& Shu, F. H. 1986, ApJ, 308, 836

Adams, F. C., Shu, F. H., \& Lada, C. J. 1988, ApJ, 326, 865

Adams, W. S., Joy, A. H., Humason, M. L.-S., \& Brayton, A. M. 1935, The spectroscopic absolute magnitudes and parallaxes of 4179 stars ([Chicago, 1935]), 11

Alexander, R. D. \& Armitage, P. J. 2006, ApJ, 639, L83

Ambartsumian, V. A. 1947, Stellar Evolution and Astrophysics (Acad. Sci. Armenian S.S.R., Erevan)

Ambartsumian, V. A. 1954, Memoires of the Societe Royale des Sciences de Liege, 1, 293

Ambartsumian, V. A. 1957, in IAU Symposium, Vol. 3, Non-stable stars, ed. G. H. Herbig, 177

Appenzeller, I. \& Dearborn, D. S. P. 1984, ApJ, 278, 689

Appenzeller, I., Oestreicher, R., \& Jankovics, I. 1984, A\&A, 141, 108

Basri, G. \& Bertout, C. 1989, ApJ, 341, 340

Beckwith, S. V. W. \& Sargent, A. I. 1991, ApJ, 381, 250

Bertout, C. 1987, in IAU Symposium, Vol. 122, Circumstellar Matter, ed. I. Appenzeller \& C. Jordan, 23-27

Bertout, C., Basri, G., \& Bouvier, J. 1988, ApJ, 330, 350

Bertout, C. \& Genova, F. 2006, A\&A, 460, 499

Bertout, C., Siess, L., \& Cabrit, S. 2007, A\&A

Burbidge, G. R. \& Burbidge, E. M. 1955, The Observatory, 75, 212

Burrows, C. J., Stapelfeldt, K. R., Watson, A. M., et al. 1996, ApJ, 473, 437

Cabrit, S., Edwards, S., Strom, S. E., \& Strom, K. M. 1990, ApJ, 354, 687

Calvet, N., Basri, G., \& Kuhi, L. V. 1984, ApJ, 277, 725

Close, L. M., Roddier, F., Northcott, M. J., Roddier, C., \& Graves, J. E. 1997, ApJ, 478, 766

Cohen, M. 1983, ApJ, 270, L69

Cohen, M. 1987, in IAU Symposium, Vol. 122, Circumstellar Matter, ed. I. Appenzeller \& C. Jordan, 39-50

Cohen, M. \& Kuhi, L. V. 1979, ApJS, 41, 743

Cram, L. E. 1979, ApJ, 234, 949

D’Alessio, P., Canto, J., Calvet, N., \& Lizano, S. 1998, ApJ, 500, 411

De Campli, W. M. 1981, ApJ, 244, 124

Dullemond, C. P., van Zadelhoff, G. J., \& Natta, A. 2002, A\&A, 389, 464

Edwards, S., Cabrit, S., Strom, S. E., et al. 1987, ApJ, 321, 473

Edwards, S. \& Snell, R. L. 1984, ApJ, 281, 237

Feigelson, E. D. \& Decampli, W. M. 1981, ApJ, 243, L89

Finkenzeller, U. \& Basri, G. 1987, in IAU Symposium, Vol. 122, Circumstellar Matter, ed. I. Appenzeller \& C. Jordan, 103-104

Ghosh, P. \& Lamb, F. K. 1979, ApJ, 232, 259

Giampapa, M. S., Calvet, N., Imhoff, C. L., \& Kuhi, L. V. 1981, ApJ, 251, 113

Greenstein, J. L. 1950, PASP, 62, 156

Gullbring, E., Hartmann, L., Briceno, C., \& Calvet, N. 1998, ApJ, 492, 323

Haro, G. 1952, ApJ, 115, 572

Haro, G. 1957, in IAU Symposium, Vol. 3, Non-stable stars, ed. G. H. Herbig, 26

Hartigan, P., Edwards, S., \& Ghandour, L. 1995, ApJ, 452, 736

Hartmann, L., Avrett, E., \& Edwards, S. 1982, ApJ, 261, 279

Hartmann, L., D'Alessio, P., Calvet, N., \& Muzerolle, J. 2006, ApJ, 648, 484

Hartmann, L. \& Kenyon, S. J. 1985, ApJ, 299, 462

Hartmann, L. \& Kenyon, S. J. 1987, ApJ, 312, 243

Herbig, G. 1974, QJRAS, 15, 526

Herbig, G. H. 1951, ApJ, 113, 697

Herbig, G. H. 1957, in IAU Symposium, Vol. 3, Non-stable stars, ed. G. H. Herbig, 3

Herbig, G. H. 1962, Advances in Astronomy and Astrophysics, 1, 47

Herbig, G. H. 1977a, ApJ, 217, 693 
Herbig, G. H. 1977b, in IAU Symposium, Vol. 75, Star Formation, ed. T. de Jong \& A. Maeder, 283

Joy, A. H. 1945, ApJ, 102, 168

Joy, A. H. 1949, ApJ, 110, 424

Joy, A. H. 1952, ApJ, 115, 25

Joy, A. H. 1954, ApJS, 1, 39

Joy, A. H. 1957, in IAU Symposium, Vol. 3, Non-stable stars, ed. G. H. Herbig, 31

Joy, A. H. 1958, PASP, 70, 505

Joy, A. H. \& Humason, M. L. 1949, PASP, 61, 133

Kenyon, S. J. \& Hartmann, L. 1987, ApJ, 323, 714

Koenigl, A. 1991, ApJ, 370, L39

Kuhi, L. V. 1964, ApJ, 140, 1409

Kuhi, L. V. 1966, PASP, 78, 430

Lada, C. J. \& Wilking, B. A. 1984, ApJ, 287, 610

Lago, M. T. V. T. 1984, MNRAS, 210, 323

Larson, R. B. 1969, MNRAS, 145, 271

Larson, R. B. 1977, in IAU Symposium, Vol. 75, Star Formation, ed. T. de Jong \& A. Maeder, $249-267$

Lynden-Bell, D. \& Pringle, J. E. 1974, MNRAS, 168, 603

Malbet, F., Lachaume, R., \& Monin, J.-L. 2001, A\&A, 379, 515

Marquis de Laplace, P.-S. 1798, Exposition du systeme du monde (Paris : V. Courcier, 1798; 551 p. ; in 4.; DCC.4.448)

Mendoza V., E. E. 1966, ApJ, 143, 1010

Mendoza V., E. E. 1968, ApJ, 151, 977

Montmerle, T., Koch-Miramond, L., Falgarone, E., \& Grindlay, J. E. 1983, ApJ, 269, 182

Mundt, R. 1987, in IAU Symposium, Vol. 122, Circumstellar Matter, ed. I. Appenzeller \& C. Jordan, $147-158$

Mundt, R. \& Fried, J. W. 1983, ApJ, 274, L83

Muzerolle, J., Hillenbrand, L., Calvet, N., Briceño, C., \& Hartmann, L. 2003, ApJ, 592, 266

Natta, A., Testi, L., Calvet, N., et al. 2007, in Protostars and Planets V, ed. B. Reipurth, D. Jewitt, \& K. Keil, 767-781

Natta, A., Testi, L., Randich, S., \& Muzerolle, J. 2005, Memorie della Societa Astronomica Italiana, 76, 343

Pinte, C., Ménard, F., Duchêne, G., \& Bastien, P. 2006, A\&A, 459, 797

Rucinski, S. M. 1985, AJ, 90, 2321

Rydgren, A. E., Strom, S. E., \& Strom, K. M. 1976, ApJS, 30, 307

Rydgren, A. E. \& Zak, D. S. 1987, PASP, 99, 141

Shakura, N. I. \& Sunyaev, R. A. 1973, A\&A, 24, 337

Shu, F. H. \& Adams, F. C. 1987, in IAU Symposium, Vol. 122, Circumstellar Matter, ed. I. Appenzeller \& C. Jordan, 7-22

Shu, F. H., Adams, F. C., \& Lizano, S. 1987, ARA\&A, 25, 23

Snell, R. L., Loren, R. B., \& Plambeck, R. L. 1980, ApJ, 239, L17

Stapelfeldt, K. R., Krist, J. E., Menard, F., et al. 1998, ApJ, 502, L65

Stapelfeldt, K. R., Ménard, F., Watson, A. M., et al. 2003, ApJ, 589, 410

Strom, K. M., Strom, S. E., Edwards, S., Cabrit, S., \& Skrutskie, M. F. 1989, AJ, 97, 1451

Strom, S. E. 1977, in IAU Symposium, Vol. 75, Star Formation, ed. T. de Jong \& A. Maeder, $179-197$

Struve, O. \& Rudkjøbing, M. 1949, ApJ, 109, 92

Ulrich, R. K. 1976, ApJ, 210, 377

Valenti, J. A., Basri, G., \& Johns, C. M. 1993, AJ, 106, 2024

Walker, M. F. 1963, AJ, 68, 298

Walker, M. F. 1972, ApJ, 175, 89

Walter, F. M. 1987, in IAU Symposium, Vol. 122, Circumstellar Matter, ed. I. Appenzeller \& C. Jordan, 107 